\title{
Genetic Diversity Analysis of Forty Pearl Millet (Pennisetum glaucum (L.) R. Br) Accessions from Sudan Using Agronomical Descriptors and DNA Molecular Markers
}

\author{
Hanaa Ibrahim Mohammed ${ }^{1}$, Nada Babiker Hamza ${ }^{2 *}$ \\ ${ }^{1}$ Agricultural Research Corporation, Biotechnology and Biosafety Research Center, Khartoum, Sudan \\ ${ }^{2}$ Commission for Biotechnology \& Genetic Engineering, National Center for Research, Khartoum, Sudan \\ Email: *nada.hamza@gmail.com
}

How to cite this paper: Mohammed, H.I. and Hamza, N.B. (2018) Genetic Diversity Analysis of Forty Pearl Millet (Pennisetum glaucum (L.) R. Br) Accessions from Sudan Using Agronomical Descriptors and DNA Molecular Markers. Advances in Bioscience and Biotechnology, 9, 322-337.

https://doi.org/10.4236/abb.2018.97021

Received: February 20, 2018

Accepted: July 17, 2018

Published: July 20, 2018

Copyright $\odot 2018$ by authors and Scientific Research Publishing Inc. This work is licensed under the Creative Commons Attribution International License (CC BY 4.0).

http://creativecommons.org/licenses/by/4.0/

cC) (7) Open Access

\begin{abstract}
Morphological descriptors and Random Amplification of Polymorphic DNA (RAPD) technique were used to assess the genetic variation among and within forty Pennesitum glaucum accessions from Sudan. Accessions were collected from 30 villages representing Darfur, North Kordofan, South Kordofan, and Blue Nile states. 64 amplified fragments were distinguished using ten primers. 63 bands were polymorphic among the forty accessions with an average of 6.3 polymorphic bands per primer. Low level of genetic similarity was observed (4\% - 43\%). The PhiPT (analogue of $\mathrm{F}_{S T}$ fixation index) value for genetic variability obtained over the four regions was 0.169 with high significance $(P=$ $0.01)$. AMOVA analysis showed higher variance components within regions $(80 \%)$ than among regions (20\%). The two dendrograms obtained by Random Amplification of Polymorphic DNA (RAPD) data; and morphological data based on 26 descriptors did not fit together. PCA (Principal coordinate's analysis) showed geographic structuring of pearl millet according to its growing regions in Sudan.
\end{abstract}

\section{Keywords}

Millet, Accessions, RAPD, Morphological, Sudan

\section{Introduction}

Pearl millet (Pennisetum glaucum (L.) R. Br.) is a cross-pollinated annual C4 crop species that originated in western Africa and was introduced to eastern Africa and Indian sub-continent some years ago [1]. India, China, Myanmar, 
Pakistan and Yemen in Asia, and Nigeria, Niger, Burkina Faso, Mali, Sudan, Chad, and Tanzania in Africa are the top countries in each region producing pearl millet grain [2].

In the western Sudan (Kordofan and Darfur), pearl millet is the stable food for the majority. For cultivation area and total production, it comes second to sorghum [3]. Sudan is the center of origin for pearl millet as it is a part of the Sahel zone which extends from Western Sudan to Senegal. About 18 species of Pennisetum are found in the country [4]. The National Gene Bank collection currently contains 1017 (pearl) millet accessions. They are maintained by the Agricultural Research Corporation in Sudan. Recently, new technologies at low scale adopting the DNA based methods were initiated [4]. Morphological characterization was the first method used by researchers to select superior genotypes, spring wheat [5], barn swallows [6], and maize [7] [8] and [9].

However, several studies showed that morphological markers are not suitable for traits with low heritability and are highly affected by environments [6] [10] [11]. These limitations have led to the evolution of molecular markers. Molecular markers can serve not only as an important tool to help in distinguishing between germplasm accessions, but also to characterize and determine genetic distance among groups of genotypes [12]. Recently, many molecular markers were used to determine the genetic relation between millet germplasms. Few researches have been done to investigate the genetic variations present in the Sudanese millet genotypes. Therefore, the main objectives of this study were to assess the genetic variation among and within forty millet accessions collected from different regions in Sudan using morphological characterization and the Random Amplified Polymorphic DNA (RAPD) technique, to study the pattern of regional genetic diversity of pearl millet, by analyzing accessions for the four studied states, and to find out whether there is a relationship between the phenotypic and genotypic characterization of studied millet accessions.

\section{Materials and Methods}

\subsection{Plant Material}

Forty millet accessions evaluated in this study were provided by the Germplasm of the Genetic Resource Unit of the Agricultural Research Corporation, Wad Medani. The seeds were collected from 30 villages from different regions of Sudan, namely: 1) North Kordofan, 2) South Kordofan, 3) Blue Nile State, and 4) Darfur (Table 1). Two Sorghum genotypes (Tabat and Wad Ahmed) were also included in the study for comparison.

\subsection{DNA Extraction}

The DNA extraction was done by CTAB 2X method. Genomic DNA was extracted from fresh leaf tissues using modified CTAB method [13]. In this method the fine powdered plant materials were immediately transferred into $13 \mathrm{ml}$ Falcon tubes containing $6 \mathrm{ml}$ of pre-warmed lysis solution. Tubes containing the 
Table 1. The pearl millet accessions used in the study and their abbreviations based on collection site.

\begin{tabular}{|c|c|c|c|c|c|}
\hline & Accession No. & $\begin{array}{c}\text { Accession } \\
\text { abbreviation }\end{array}$ & & Accession No. & $\begin{array}{c}\text { Accession } \\
\text { abbreviation }\end{array}$ \\
\hline 1 & HSD 3509 & NI & 21 & HSD 6080 & S21 \\
\hline 2 & HSD 3510 & $\mathrm{~N} 2$ & 22 & HSD 6081 & S22 \\
\hline 3 & HSD 3512 & N3 & 23 & HSD 6082 & S23 \\
\hline 4 & HSD 3517 & N4 & 24 & HSD 2369 & S24 \\
\hline 5 & HSD 3522 & N5 & 25 & HSD 5536 & B25 \\
\hline 6 & HSD 3523 & N6 & 26 & HSD 5546 & B26 \\
\hline 7 & HSD 3524 & N7 & 27 & HSD 5551 & B27 \\
\hline 8 & HSD 3525 & N8 & 28 & HSD 5558 & B28 \\
\hline 9 & HSD 3528 & N9 & 29 & HSD 5560 & B29 \\
\hline 10 & HSD 4263 & S10 & 30 & HSD 5564 & B30 \\
\hline 11 & HSD 4265 & S11 & 31 & HSD 5566 & B31 \\
\hline 12 & HSD 4269 & S12 & 32 & HSD 5574 & B32 \\
\hline 13 & HSD 4271 & S13 & 33 & HSD 5581 & B33 \\
\hline 14 & HSD 4272 & S14 & 34 & HSD 2090 & D34 \\
\hline 15 & HSD 4277 & S15 & 35 & HSD 2132 & D35 \\
\hline 16 & HSD 4283 & S16 & 36 & HSD 2150 & D36 \\
\hline 17 & HSD 4285 & S17 & 37 & HSD 2157 & D37 \\
\hline 18 & HSD 4286 & S18 & 38 & HSD 2168 & D38 \\
\hline 19 & HSD 6073 & S19 & 39 & HSD 2223 & D39 \\
\hline 20 & HSD 6076 & S20 & 40 & HSD 2245 & D40 \\
\hline
\end{tabular}

$\mathrm{N}=$ North Kordofan, $\mathrm{S}=$ South Kordofan, $\mathrm{B}=$ Blue Nile State, $\mathrm{D}=$ Darfur.

samples were then incubated in water path at $65^{\circ} \mathrm{C}$ with gentle shaking for 30 minutes and left to cool at room temperature for $5 \mathrm{~min}$. Isoamyl alcohol and chloroform mixture $(1: 24)$ was added to each tube and the phases were mixed gently for $5 \mathrm{~min}$. at room temperature to make a homogenous mixture. The cell debris was removed by centrifugation at $5000 \mathrm{rpm}$ for $15 \mathrm{~min}$ at room temperature and the resulted clear aqueous phases (containing DNA) were transferred to new sterile tubes. The step of the chloroform: Isoamyl alcohol extraction was repeated twice. The nucleic acid in the aqueous phase was precipitated by adding equal volume of deep cooled isopropanol. The contents were mixed gently and collected by centrifugation at $4000 \mathrm{rpm}$ for $10 \mathrm{~min}$. Then the formed DNA pellet was washed twice with $70 \%$ ethanol and the ethanol was discarded after spinning with flash centrifugation the remained ethanol was removed by leaving the pellet to dry at room temperature. The pellet was dissolved in TE buffer and stored at $-20^{\circ} \mathrm{C}$ for further use. The extracted DNA samples were observed under UV illumination after staining with ethidium bromide and agarose gel electrophoresis. 


\subsection{RAPD Analysis and Primer Selection}

Twenty primers were used for PCR amplification, ten primers that produced strongly amplified polymorphic bands with these test templates were selected for RAPD-PCR analysis (Table 2). The PCR reaction mixtures were prepared in 25 $\mu \mathrm{l}$ volumes containing $2.5 \mu \mathrm{l}$ of $10 \mathrm{X}$ Taq buffer, $1.5 \mu \mathrm{lgCl}_{2}(50 \mathrm{mM}), 2.5 \mu \mathrm{l}$ dNTPs $(2 \mathrm{Mm} / \mu \mathrm{l}), 2 \mu \mathrm{l}$ random primer $(10 \mathrm{pmol} / \mu \mathrm{l}), 0.5 \mu \mathrm{l}$ Taq DNA polymerase $(5 \mathrm{U} / \mu \mathrm{l})$ and $1 \mu \mathrm{l}$ of the extracted DNA (10 ng). The mixture was made up to $25 \mu$ by addition of $\mathrm{DDH}_{2} \mathrm{O}$ (double sterilized distilled water). RAPD/PCR reactions were optimized and initiated using an applied Biometra thermalcycler programmed to repeat the thermal profile. Setting of the PCR program is based on three steps. Step one, was an initial denaturation step at $94^{\circ} \mathrm{C}$ for 5 mins. Step two, was run for 40 cycles, each starting with denaturation at $94^{\circ} \mathrm{C}$ for $1 \mathrm{~min}$, followed by annealing $36^{\circ} \mathrm{C}$ for $1 \mathrm{~min}$ and ended by extension at $72^{\circ} \mathrm{C}$ for $1 \mathrm{~min}$. Step three, was a final extension cycle performed at $72^{\circ} \mathrm{C}$ for 7 minutes. The PCR machine was adjusted to hold the product at $4^{\circ} \mathrm{C}$. The PCR product was mixed with $3 \mu \mathrm{l}$ of loading dye and spun briefly in a micro centrifuge before loading. The PCR products and $1 \mathrm{~kb}$ DNA ladder were electrophoresed using 2\% agarose gel at 100 volts followed by ethidium bromide then separated fragments and were visualized with ultraviolet (UV) transilluminator (Figure 1).

\subsection{Analysis of Genetic Diversity}

Each band in the RAPDs profile was treated as an independent locus with two alleles. The numbers of bands produced for each primer were scored manually for presence (1), or absence (0) and a binary matrix was generated and then used for further analysis [14]. The PhiPT (analogue of $\mathrm{F}_{S T}$ fixation index) value for genetic variability was calculated for the four regions using GenALEx v.6.1 [15]. With AMOVA, the variance components and their significance levels for variation
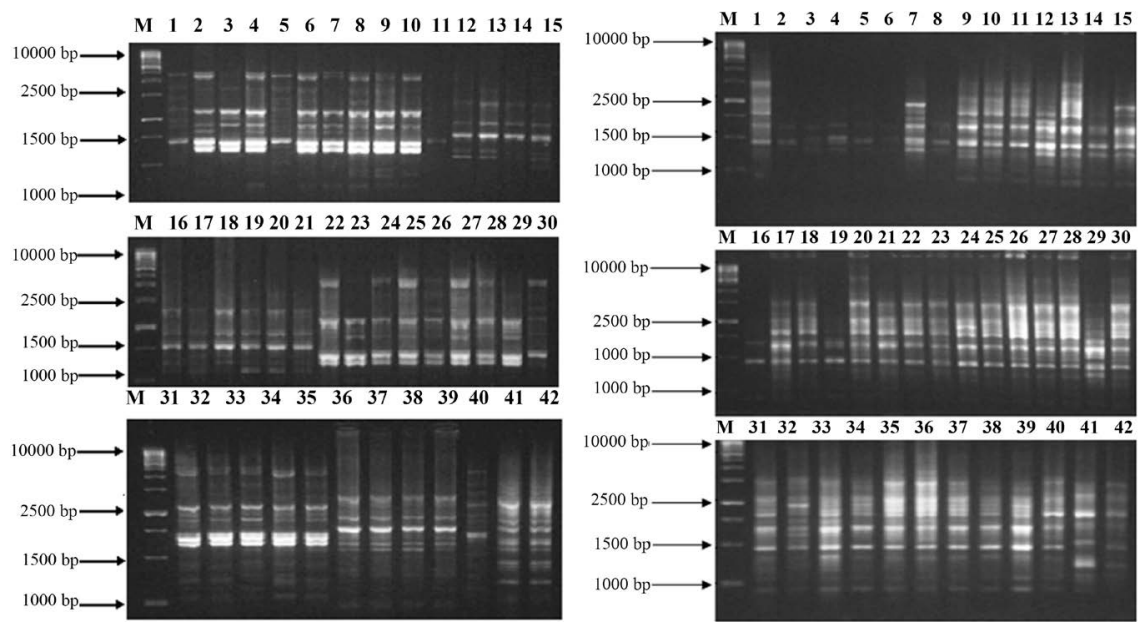

Figure 1. RAPD amplification patterns with primers D-8 and UBC-104 (from left to right: $\mathrm{M}=$ ladder, lanes 1 to 40 represent the millet accessions studied, and lanes 41 and 42 are sorghum genotypes. 
Table 2. Polymorphism detected by the use of ten polymorphic random primers on forty Pennisetum glaucum accessions.

\begin{tabular}{cccccc}
\hline $\begin{array}{c}\text { Name of } \\
\text { primer }\end{array}$ & $\begin{array}{c}\text { Sequence of primer } \\
\left(5^{\prime} \text { to } 3^{\prime}\right)\end{array}$ & $\begin{array}{c}\text { Total number } \\
\text { of bands }\end{array}$ & $\begin{array}{c}\text { Number of } \\
\text { polymorphic } \\
\text { bands }\end{array}$ & $\begin{array}{c}\text { Number of } \\
\text { monomorphic } \\
\text { bands }\end{array}$ & $\begin{array}{c}\text { \% of } \\
\text { polymorphic } \\
\text { bands }\end{array}$ \\
\hline A-1 & CAG GCC CTT C & 7 & 7 & 0 & 100 \\
B-8 & GTC CAC ACG G & 10 & 10 & 0 & 100 \\
B-20 & GGA CCC TTA C & 9 & 9 & 0 & 100 \\
C-2 & GTG AGG CGT C & 6 & 6 & 0 & 100 \\
C-10 & TGT CTG GGT G & 6 & 6 & 0 & 100 \\
D-8 & GTG TGC CCC A & 6 & 6 & 0 & 100 \\
UBC-101 & GCG GCT GGA G & 4 & 3 & 1 & 75 \\
UBC-104 & GGG CAA TGA T & 5 & 5 & 0 & 100 \\
UBC-122 & GTA GAC GAG C & 5 & 5 & 0 & 100 \\
UBC-155 & CTG GCG GCT G & 6 & 6 & 0 & 100 \\
Total & & 64 & 63 & 1 & 975 \\
Average & & 6.4 & 6.3 & 0.1 & 97.5 \\
\hline
\end{tabular}

among regions and within regions were obtained by using RAPD data of the 40 accessions (Table 3) and (Figure 2).

\subsection{Genetic Structure}

A principal coordinate analysis (PCA) was conducted with GenALEx v.6.1. This multivariate approach was chosen to complement the cluster analysis information (Figure 3), because cluster analysis is more sensitive to closely related individuals whereas PCA is more informative regarding distances among major groups [16].

Pairwise genetic distance between individuals were calculated by the percentage disagreement method (Table 4). These data were used in cluster analysis with the unweighted pair-group method using arithmetic averages (UPGMA), in which samples were grouped based on their similarity (Figure 4) with the aid of statistical software package STATISTCA-ver.6 [17].

\subsection{Agronomical Field Study}

Phenotypic diversity and agronomic descriptors for the 40 millet accessions were also recorded based on 26 agronomical descriptors for each accession. These observed data were taken during the season 2006-2007 (Table 5).

\subsection{Statistical Analysis of Agronomical Descriptors}

Analysis of variance was done using the SPSS version 17 computer software [18] program in order to assort accessions according to their agronomic characters (Figure 5). 
Table 3. Summary of the analysis of molecular variance (AMOVA) within and among the four Pennisetum glaucum regions in Sudan.

\begin{tabular}{lcccccccc}
\hline Source & Df & SS & MS & Est. Var. & $\%$ & Stat & Value & Probability \\
\hline $\begin{array}{l}\text { Among } \\
\text { regions }\end{array}$ & 3 & 87.584 & 29.195 & 2.116 & $20 \%$ & & & \\
$\begin{array}{l}\text { Within } \\
\text { regions }\end{array}$ & 36 & 312.216 & 8.673 & 8.673 & $80 \%$ & PhiPT & 0.196 & 0.010 \\
Total & 39 & 399.800 & & 10.788 & $100 \%$ & & & \\
\hline
\end{tabular}

The analysis is based on RAPD phenotypes consisting of 63 bands states.

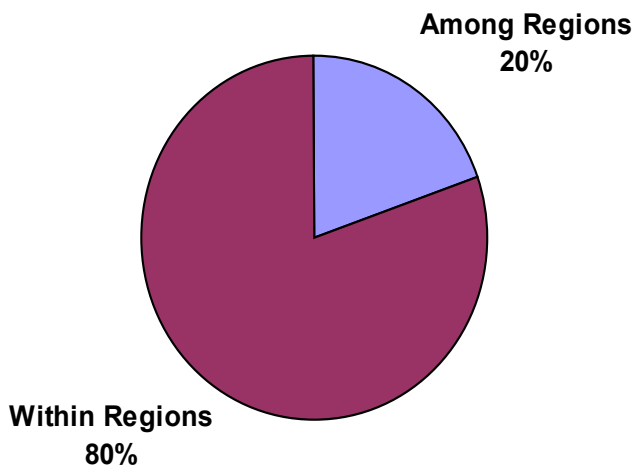

Figure 2. Percentages of molecular variance within and among the four Pennisetum glaucum regions based on 63 RAPD loci.

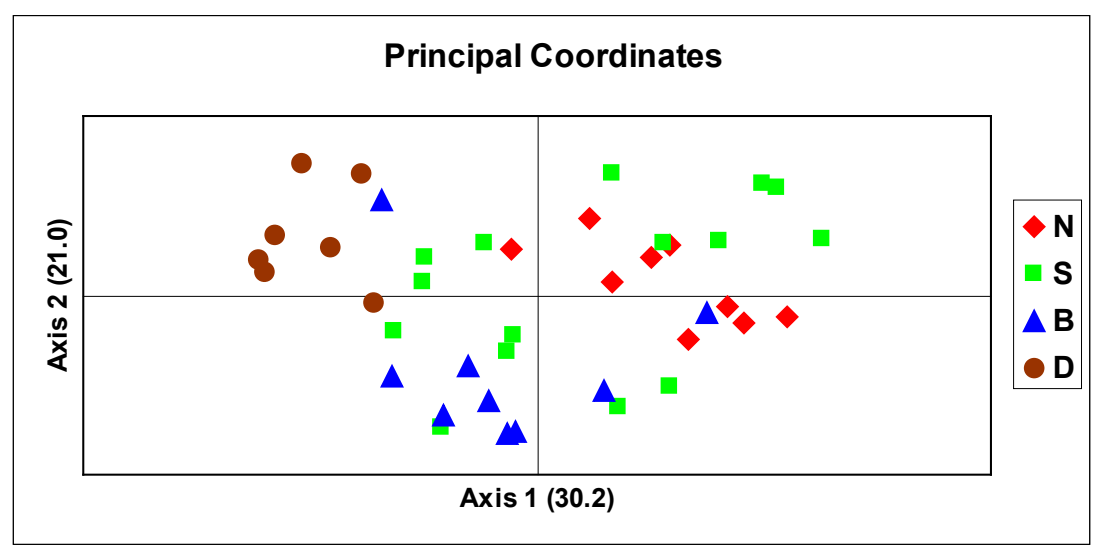

Figure 3. Principal coordinates analysis for the four Pennisetum glaucum regions. Note the separation between Darfur individuals and the other three regions; where $\mathrm{N}=$ North Kordofan, $\mathrm{S}=$ South Kordofan, $\mathrm{B}=$ Blue Nile and $\mathrm{D}=$ Darfour.

\section{Results}

\subsection{Amplification of RAPD Primers}

Ten primers which were informative (UBC-101, UBC-104, UBC-122, UBC-155, A-1, B-8, B-20, C-2, C-10, D-8) were selected and used to evaluate the degree of polymorphism and genetic relationship within and between all individuals under study (Table 2). The selected primers generated an appropriate amplification pattern with clear and consistent reproducible bands. The maximum 


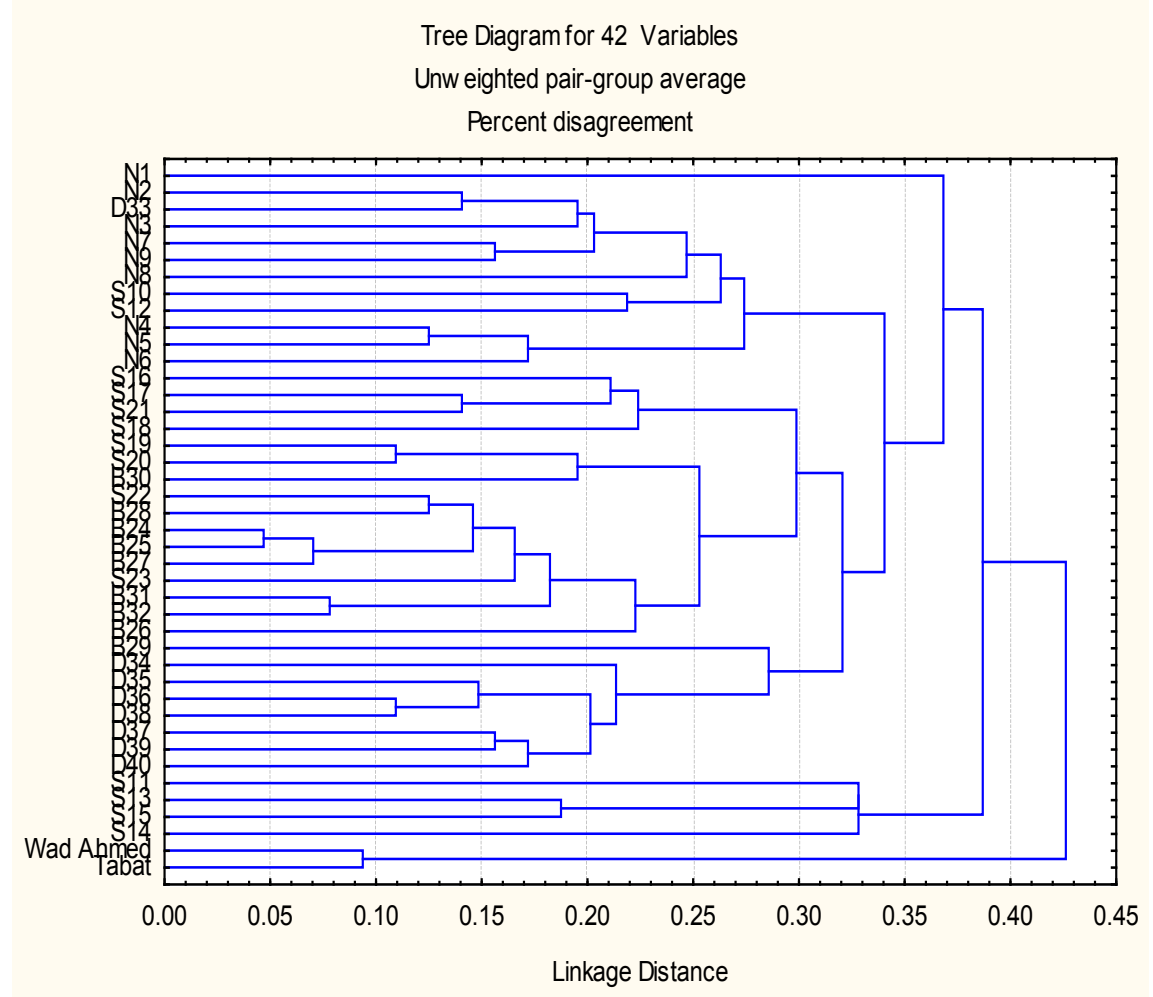

Figure 4. Dendrogram based on UPGMA clustering using the percentage disagreement, showing relationships of 40 pearl millet accessions of and two genotypes of Sorghum. Where $\mathrm{N}=$ North Kordofan, $\mathrm{S}=$ South Kordofan, B = Blue Nile and D = Darfour.

Table 4. Matrix of RAPD dissimilarity among 40 Pennisetum glaucum Accessions based on Nei and Lei coefficients.

$1 \quad 2 \quad 3 \quad 4 \quad 5 \quad 6 \quad 7 \quad 8 \quad 9 \quad 101112131415161718192021222324252627282930313233 \quad 343536373839404142$

$1 \dot{8}$

$2 \stackrel{\circ}{\stackrel{\circ}{\mathrm{N}}} \dot{8}$

$3 \underset{\omega}{\stackrel{0}{0}} \stackrel{\circ}{i} \dot{8}$

$4 \underset{\infty}{\circ} \stackrel{\circ}{\circ} \stackrel{0}{\dot{N}} \dot{8}$

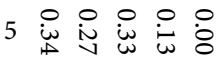

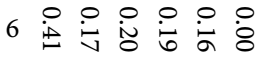

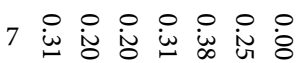

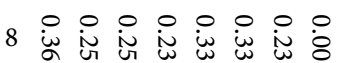

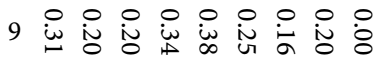

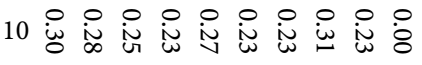


H. I. Mohamed, N. B. Hamza

Continued

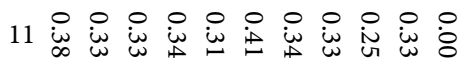

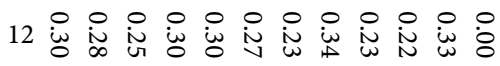

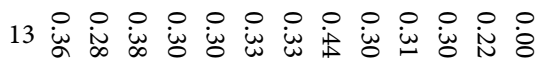

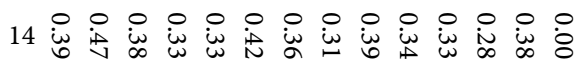

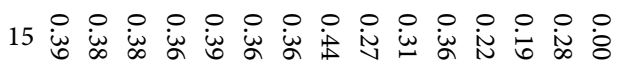

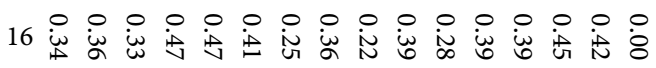

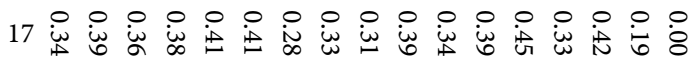

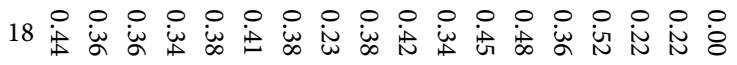

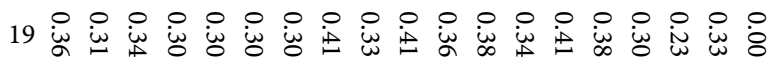

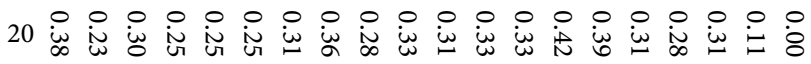

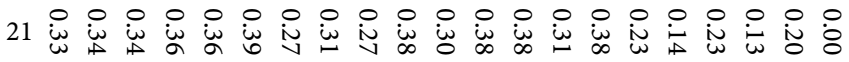

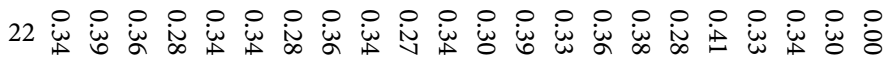

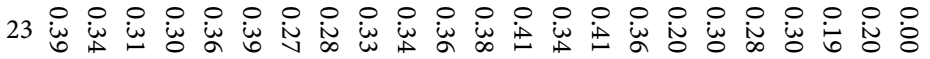

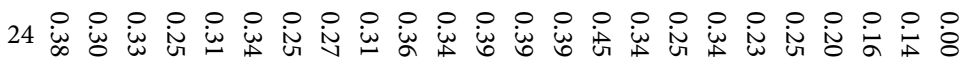

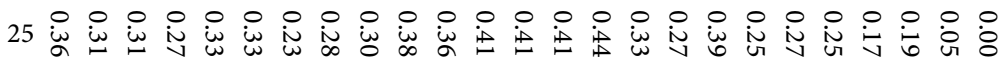

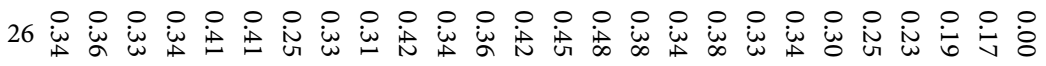

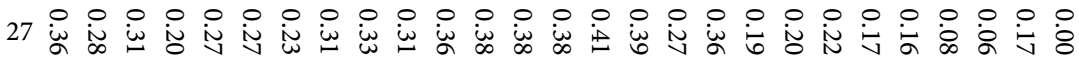

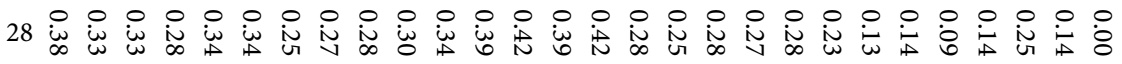

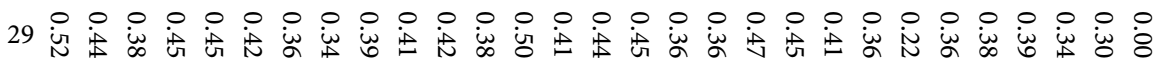

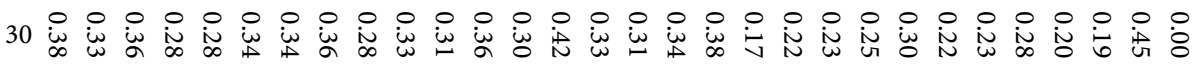

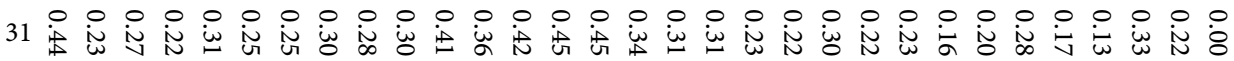

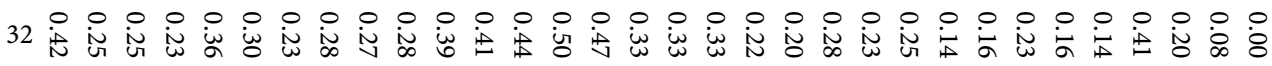

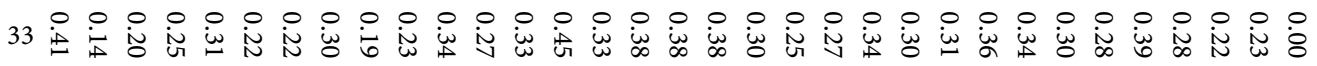

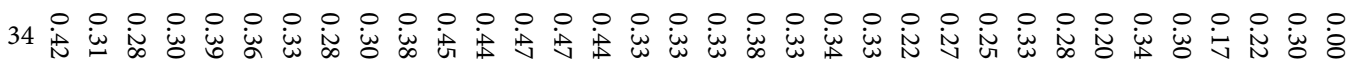

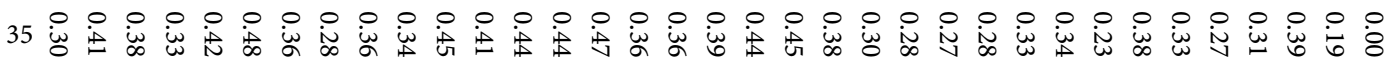

DOI: 10.4236/abb.2018.97021

329

Advances in Bioscience and Biotechnology 


\section{Continued}

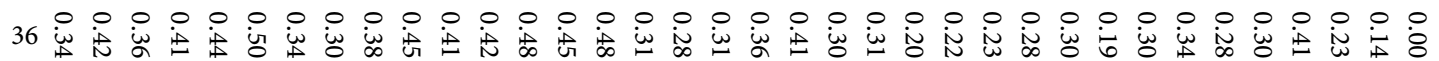

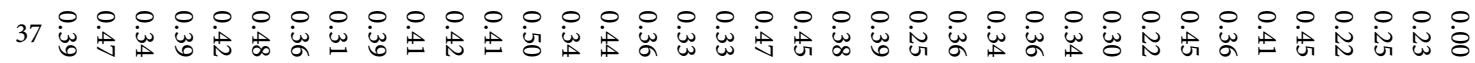

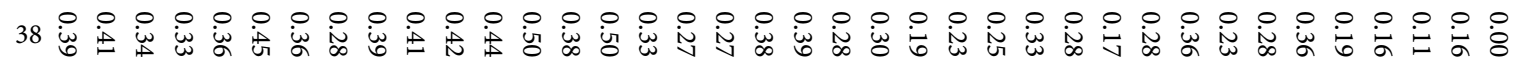

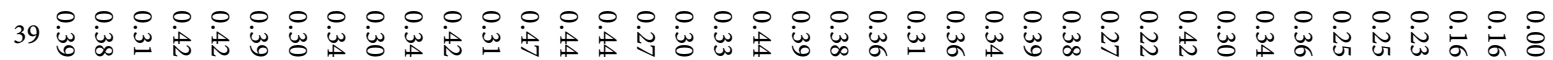

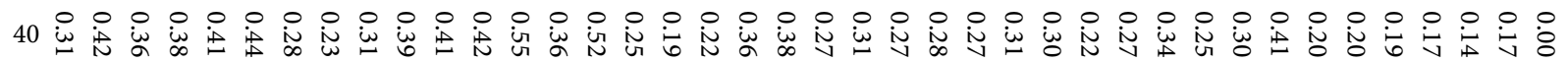

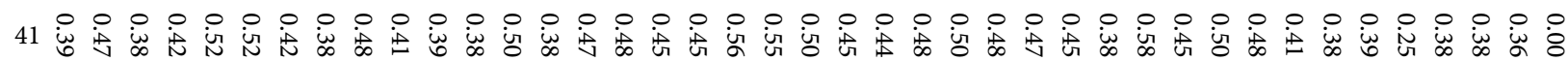

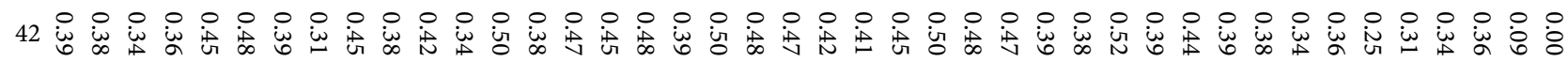

Table 5. The 26 morpho-agronomical parameters taken from field experiment during season 2006-2007.

\begin{tabular}{|c|c|c|c|c|c|c|c|c|c|c|c|c|c|c|c|c|c|c|c|c|c|c|c|c|c|c|}
\hline $\begin{array}{c}\text { Sample } \\
\text { No }\end{array}$ & A & B & C & D & E & $\mathrm{F}$ & $\mathrm{G}$ & $\mathrm{H}$ & I & $\mathrm{J}$ & K & $\mathrm{L}$ & $\mathrm{M}$ & $\mathrm{N}$ & $\mathrm{O}$ & $\mathrm{P}$ & Q & $\mathrm{R}$ & $S$ & $\mathrm{~T}$ & $\mathrm{U}$ & $\mathrm{V}$ & $\mathrm{W}$ & $\mathrm{X}$ & $\mathrm{Y}$ & $\mathrm{Z}$ \\
\hline N1 & 228 & 4.4 & 3 & 6 & 1 & 3 & 4 & 3 & 3 & 4 & 4 & 2 & 68 & 4 & 3 & 2.5 & 25.5 & 3 & 2 & 3 & 4 & 9 & 2 & 3 & 4 & 9.1 \\
\hline N2 & 222 & 4.5 & 3 & 5 & 3 & 3 & 4 & 4 & 4 & 3 & 4 & 2 & 81 & 4 & 3 & 1.5 & 28.5 & 2.5 & 2 & 2 & 4 & 10 & 3 & 3 & 4 & 9 \\
\hline N3 & 219 & 5 & 2 & 6 & 2 & 4 & 4 & 3 & 3 & 4 & 4 & 3 & 76 & 4 & 3 & 4.6 & 31 & 3.5 & 5 & 2 & 4 & 11 & 2 & 3 & 4 & 8.4 \\
\hline $\mathrm{N} 4$ & 211 & 3.2 & 2 & 6 & 2 & 2 & 2 & 4 & 4 & 3 & 2 & 2 & 60 & 4 & 3 & 16.3 & 26.5 & 2.9 & 7 & 2 & 4 & 8 & 3 & 3 & 4 & 9.1 \\
\hline N5 & 238 & 4.4 & 3 & 3 & 1 & 4 & 4 & 3 & 4 & 3 & 4 & 2 & 69 & 4 & 3 & 7.5 & 35.3 & 3.5 & 5 & 2 & 3 & 12 & 2 & 3 & 4 & 10.8 \\
\hline N6 & 207 & 3.5 & 2 & 4 & 1 & 3 & 3 & 4 & 4 & 3 & 4 & 2 & 77 & 2 & 3 & 1 & 31.5 & 3 & 5 & 3 & 4 & 9 & 2 & 4 & 4 & 10.4 \\
\hline N7 & 218 & 5 & 3 & 3 & 1 & 2 & 3 & 3 & 4 & 4 & 4 & 2 & 71 & 4 & 3 & 10.5 & 29 & 3 & 7 & 3 & 2 & 11 & 2 & 3 & 4 & 12.3 \\
\hline N8 & 233 & 4 & 2 & 5 & 2 & 4 & 4 & 3 & 3 & 3 & 2 & 2 & 62 & 2 & 3 & 4 & 33.5 & 3.8 & 7 & 3 & 4 & 12 & 2 & 3 & 4 & 12.3 \\
\hline N9 & 221 & 5.4 & 3 & 4 & 2 & 3 & 3 & 3 & 4 & 3 & 4 & 2 & 69 & 4 & 3 & 3.5 & 25 & 3.5 & 7 & 4 & 2 & 12 & 2 & 3 & 4 & 11.4 \\
\hline S10 & 235 & 5 & 3 & 5 & 1 & 3 & 4 & 4 & 4 & 4 & 4 & 2 & 74 & 2 & 3 & 10.3 & 23 & 2.9 & 2 & 2 & 2 & 12 & 2 & 4 & 4 & 8 \\
\hline S11 & 210 & 4 & 4 & 5 & 1 & 3 & 4 & 4 & 4 & 3 & 4 & 2 & 65 & 4 & 3 & 5 & 31 & 2.8 & 1 & 3 & 4 & 12 & 2 & 3 & 4 & 12.3 \\
\hline $\mathrm{S} 12$ & 253 & 4.4 & 2 & 3 & 1 & 4 & 4 & 4 & 4 & 3 & 4 & 2 & 77 & 2 & 3 & 4.5 & 36.5 & 2.3 & 3 & 2 & 2 & 9 & 2 & 3 & 4 & 8.6 \\
\hline $\mathrm{S} 13$ & 200 & 3.4 & 2 & 2 & 2 & 4 & 4 & 3 & 4 & 4 & 4 & 2 & 77 & 4 & 3 & 8.4 & 24 & 3 & 2 & 3 & 4 & 11 & 2 & 3 & 4 & 13.6 \\
\hline S14 & 282 & 4.4 & 3 & 3 & 2 & 4 & 4 & 3 & 4 & 3 & 4 & 2 & 76 & 4 & 3 & 11.5 & 26 & 2.5 & 1 & 3 & 2 & 13 & 2 & 3 & 4 & 10.5 \\
\hline S15 & 267 & 5 & 3 & 3 & 1 & 2 & 4 & 3 & 3 & 4 & 3 & 3 & 77 & 4 & 3 & 16.5 & 32 & 3.3 & 3 & 3 & 2 & 12 & 2 & 3 & 4 & 12 \\
\hline S16 & 185 & 4.4 & 2 & 4 & 1 & 3 & 3 & 4 & 3 & 3 & 4 & 3 & 70 & 4 & 3 & 8 & 25 & 2 & 3 & 2 & 4 & 9 & 3 & 3 & 4 & 8.5 \\
\hline S17 & 200 & 3 & 3 & 3 & 1 & 2 & 4 & 4 & 4 & 3 & 3 & 4 & 82 & 4 & 4 & 0 & 32 & 4 & 3 & 2 & 2 & 12 & 2 & 3 & 4 & 7.6 \\
\hline S18 & 231 & 5 & 3 & 5 & 2 & 3 & 3 & 4 & 3 & 3 & 3 & 2 & 80 & 4 & 3 & 4.5 & 33 & 2.3 & 3 & 4 & 3 & 11 & 2 & 4 & 4 & 9.8 \\
\hline S19 & 212 & 5.4 & 2 & 4 & 2 & 2 & 3 & 3 & 3 & 3 & 3 & 2 & 80 & 4 & 3 & 3.5 & 26 & 3.3 & 2 & 3 & 2 & 10 & 2 & 3 & 4 & 8.5 \\
\hline S20 & 237 & 4 & 2 & 3 & 1 & 3 & 3 & 4 & 3 & 4 & 4 & 2 & 68 & 4 & 3 & 5.4 & 31 & 2 & 3 & 2 & 4 & 11 & 2 & 3 & 4 & 9.1 \\
\hline S21 & 279 & 4 & 3 & 6 & 2 & 2 & 3 & 3 & 4 & 3 & 4 & 2 & 85 & 3 & 3 & 4 & 33 & 2.8 & 3 & 2 & 4 & 13 & 3 & 3 & 4 & 7.3 \\
\hline S22 & 279 & 6 & 4 & 4 & 1 & 2 & 4 & 4 & 3 & 3 & 3 & 2 & 72 & 4 & 4 & 0 & 35.5 & 3.5 & 3 & 3 & 2 & 14 & 2 & 3 & 3 & 9.3 \\
\hline S23 & 205 & 5 & 3 & 4 & 2 & 2 & 4 & 3 & 4 & 4 & 4 & 2 & 80 & 4 & 3 & 6 & 32 & 3 & 3 & 3 & 2 & 11 & 2 & 3 & 3 & 8.2 \\
\hline S24 & 266 & 5.4 & 3 & 3 & 1 & 2 & 4 & 4 & 4 & 3 & 3 & 2 & 82 & 4 & 4 & 0 & 33 & 2.5 & 3 & 3 & 4 & 14 & 2 & 3 & 4 & 9 \\
\hline B25 & 289 & 4.4 & 2 & 2 & 2 & 3 & 3 & 3 & 4 & 3 & 4 & 2 & 80 & 4 & 3 & 5.5 & 28.5 & 2.5 & 1 & 3 & 2 & 11 & 2 & 3 & 3 & 11.6 \\
\hline B26 & 237 & 4 & 2 & 3 & 2 & 2 & 4 & 2 & 4 & 3 & 4 & 3 & 77 & 3 & 4 & 0 & 31 & 2.5 & 2 & 2 & 4 & 11 & 3 & 3 & 4 & 8.4 \\
\hline B27 & 238 & 4 & 3 & 4 & 1 & 2 & 4 & 4 & 4 & 3 & 4 & 2 & 77 & 4 & 3 & 5.5 & 37 & 3.6 & 4 & 3 & 4 & 12 & 2 & 3 & 4 & 9.1 \\
\hline
\end{tabular}




\begin{tabular}{|c|c|c|c|c|c|c|c|c|c|c|c|c|c|c|c|c|c|c|c|c|c|c|c|c|c|c|}
\hline \multicolumn{27}{|c|}{ Continued } \\
\hline B28 & 237 & 4 & 3 & 2 & 1 & 4 & 3 & 4 & 4 & 4 & 4 & 2 & 67 & 4 & 3 & 13.8 & 32 & 2.5 & 1 & 3 & 4 & 12 & 2 & 3 & 4 & 11.8 \\
\hline B29 & 305 & 4.4 & 3 & 2 & 2 & 2 & 3 & 3 & 4 & 3 & 4 & 2 & 76 & 4 & 3 & 1.8 & 34 & 2.5 & 1 & 4 & 2 & 11 & 3 & 3 & 4 & 10.2 \\
\hline B30 & 215 & 4.4 & 3 & 4 & 2 & 2 & 3 & 4 & 3 & 4 & 3 & 2 & 67 & 4 & 3 & 6.4 & 28 & 2.8 & 7 & 3 & 2 & 11 & 2 & 3 & 4 & 10.3 \\
\hline B31 & 179 & 5 & 2 & 3 & 2 & 4 & 3 & 4 & 4 & 3 & 4 & 2 & 70 & 3 & 3 & 10.9 & 35 & 3.5 & 3 & 3 & 4 & 10 & 2 & 4 & 4 & 11.8 \\
\hline B32 & 194 & 4 & 2 & 2 & 1 & 4 & 2 & 4 & 3 & 4 & 4 & 4 & 70 & 2 & 3 & 8,4 & 24 & 3 & 1 & 2 & 4 & 12 & 3 & 3 & 4 & 15.1 \\
\hline B33 & 217 & 4.4 & 2 & 2 & 2 & 2 & 3 & 2 & 3 & 3 & 3 & 3 & 65 & 4 & 3 & 5 & 31 & 2.5 & 2 & 3 & 4 & 10 & 3 & 3 & 4 & 14.3 \\
\hline D34 & 234 & 4.4 & 4 & 2 & 2 & 2 & 3 & 4 & 4 & 3 & 4 & 2 & 68 & 3 & 3 & 9.5 & 28.5 & 2.5 & 5 & 3 & 2 & 7 & 3 & 3 & 3 & 9 \\
\hline D35 & 255 & 4.4 & 4 & 2 & 2 & 2 & 4 & 4 & 4 & 4 & 4 & 2 & 75 & 4 & 3 & 10.4 & 28.5 & 3.8 & 6 & 2 & 4 & 13 & 3 & 3 & 4 & 13.5 \\
\hline D36 & 200 & 4 & 2 & 5,6 & 2 & 3 & 4 & 4 & 3 & 4 & 3 & 2 & 75 & 4 & 3 & 0.3 & 15,22 & $3,2.5$ & 7 & 3 & 3 & 10 & 3 & 3 & 4 & 10.6 \\
\hline D37 & 237 & 5 & 4 & 6 & 2 & 3 & 3 & 4 & 3 & 3 & 1 & 2 & 65 & 4 & 3 & 9 & 29 & 2 & 1 & 4 & 2 & 10 & 4 & 3 & 4 & 9.5 \\
\hline D38 & 270 & 3.4 & 3 & 2 & 1 & 4 & 4 & 3 & 4 & 4 & 4 & 2 & 77 & 4 & 3 & 1 & 22.5 & 3.3 & 1 & 3 & 4 & 15 & 2 & 3 & 4 & 9.1 \\
\hline D39 & 257 & 4.4 & 4 & 5 & 2 & 3 & 2 & 4 & 4 & 4 & 3 & 2 & 77 & 4 & 4 & 10 & 35.5 & 3.3 & 6 & 4 & 2 & 15 & 3 & 3 & 4 & 9.1 \\
\hline D40 & 255 & 4 & 3 & 5 & 2 & 2 & 3 & 4 & 4 & 3 & 4 & 2 & 83 & 4 & 4 & 5 & 33 & 3 & 3 & 3 & 4 & 13 & 2 & 3 & 4 & 9 \\
\hline
\end{tabular}

$\mathrm{A}=$ Plant height $(\mathrm{cm}), \mathrm{B}=$ Stem diameter $(\mathrm{cm}), \mathrm{C}=$ Early vigour, $\mathrm{D}=$ Total no. of tillers, $\mathrm{E}=$ no. of productive tillers, $\mathrm{F}=$ No. of Nodal tillers, $\mathrm{G}=\mathrm{Plant}$ aspect, $\mathrm{I}=$ Green fodder yield potential, $\mathrm{J}=$ Spike shape, $\mathrm{K}=$ Spikelet shattering, $\mathrm{L}=$ Bristle length, $\mathrm{M}=$ Days to $50 \%$ flowering, $\mathrm{N}=\mathrm{Flowering}$ range, $\mathrm{O}=$ Ear exertion type, $\mathrm{P}=$ Ear exertion distance $(\mathrm{cm}), \mathrm{Q}=$ Spike length $(\mathrm{cm}), \mathrm{R}=$ Spike thickness $(\mathrm{cm}), \mathrm{S}=$ Seed color, $\mathrm{T}=$ Seed covering, $\mathrm{U}=$ Seed shape, $\mathrm{V}=$ No. of leaves, $\mathrm{W}=$ Senescence, $\mathrm{X}=$ Juice quality, $\mathrm{Y}=$ Stalk Juiciness, $\mathrm{Z}=1000$ Seed weight $(\mathrm{g})$.

number of fragments was produced by the primer B-8 (10 bands) with $100 \%$ polymorphism while the minimum number of fragments was produced by the primer UBC-101 (4 bands) with 75\% polymorphism. A total of 64 amplified fragments were distinguished across the selected primers and the statistical analysis showed 63 polymorphic bands among the 40 accessions with an average of 6.3 polymorphic bands per primer and only one monomorphic band with an average 0.1 . The number of bands per primer ranged from 4 to 10 and the number of polymorphic bands per primer varied from 3 to 10 . All the primers which were used in this study were $100 \%$ polymorphic, except primer UBC-101 which was $75 \%$ polymorphic (Table 2 ).

The analysis of molecular variance (AMOVA) for the four regions showed significant difference $(P<0.01)$, with $80 \%$ of the differentiation attributed to within regions and $20 \%$ attributed to among regions (Figure 2).

The PhiPT estimates were moderate to high $(0.196)$ and significant $(P=0.01)$ (Table 3). The UPGMA and percent disagreement values (PDV) were used to estimate the degree of relationships between the individuals analyzed based on common amplified fragments. Based on the matrix obtained (Table 4), the percent disagreement values (PDV) for all individuals was ranging from $4 \%$ to $43 \%$. Principal components analysis of molecular variance performed in GenAlex 6.1 was based on individual banding pattern.

\subsection{Agronomical Analysis}

The cluster analysis based on Euclidean distance (Figure 5) using agronomical characters grouped the forty pearl millet accessions into two main clusters. B32 was alone in a separate arm as the most divergent accession. Cluster one included 

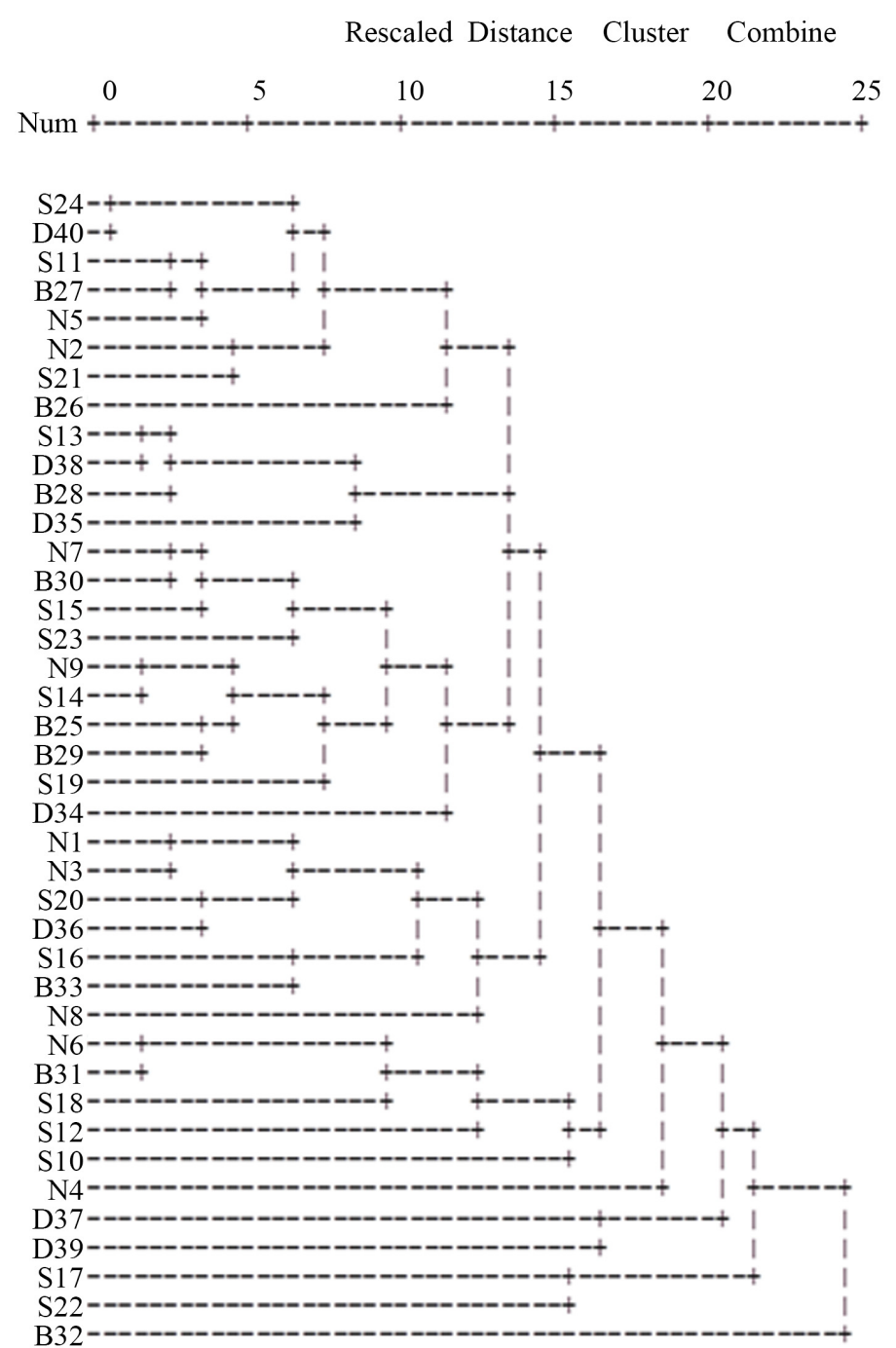

Figure 5. Cluster diagram based on SPSS analysis for the 40 pearl millet accessions classified using 26 agronomic characters.

two accessions S17 and S22, cluster two divided into two subgroups, subgroup one includes two accessions and subgroup two divided into many sub-clusters. The evaluation of pearl millet accessions based on the 26 agronomical descriptors had shown that there is a high amount of variation among the accessions.

\section{Discussion}

\subsection{Molecular Analysis}

The genetic similarity obtained in this study ranged from $4 \%$ to $43 \%$. These results are in agreement with other results reported by [12] who used 32 RAPD primers for 11 pearl millet accessions from different commercial agricultural areas (the Cerrados region of Brazil). They found that polymorphism among the pearl millet accessions tested, ranged from $9 \%$ to $76 \%$. In the first cluster of the dendrogram, it is clear that millet accessions from Darfour except D33, Blue Nile 
and most millet accessions from South Kordofan grouped together. However, within this group, Darfour millet genotypes are clearly distinct as they fall in a separate sub-group and accessions from Blue Nile and South Kordofan fall in another sub group. All North Kordofan accessions fall in a second group containing only two accessions from South Kordofan and one accession from Darfour. The close genetic similarity which exhibited by accessions from different regions (Blue Nile and Darfur, Blue Nile and South Kordofan) might be as a result movement of seeds among these regions. The fact that it is very difficult for farmers to keep their own selected seeds and the consequent substantial seed exchanges between them is probably the main factor accounting for the genetic homogeneity which has been observed in this study. South Kordofan accessions were the most heterogeneous genotypes based on their scattered positions in the dendrogram and their highest percentage of polymorphic (88.2\%). Whereas, Darfour genotypes were the most distinct millet accessions. So results from this study can help breeding programs and samples selections can become very easy, quick and useful for the millet breeders. The genetic "similarity" between the significant fraction of the South Kordofan pearl millet accessions and the Blue Nile accessions could illustrate a preferential seed flow. It is likely that South Kordofan region is a provider of seed lots to Blue Nile region that can be approved by the higher polymorphism found in South Kordofan region (88.2\%), whereas Blue Nile maintains (68.7\%) as shown from the regional diversity analysis. This illustrates a significant seed flow. PhiPT and $\mathrm{F}_{S T}$ are analogous standardizing measures of the degree of genetic differentiation among regions: scores for both measures range from 0 (non differentiation) to 1 (non alleles shared). The Principle Coordinate Analysis for the four Pennisetum glaucum regions showed clear scattering of the South Kordofan genotypes and all the other three regions. However, it was also clear that Darfur genotypes remained intact in a distant group. In this study, the PhiPT value obtained over all regions was (0.196) with high significance $(P=0.01)$, suggesting tendency to high structuring within the region. There was genetic variation of $80 \%$ within the regions and the estimated genetic variation was $20 \%$ among them. Every region still maintains its indigenous genetic material so that these farmers keep their own seeds sources. So the pattern that is depicted by the PCA analysis is in consistence with the UPGMA tree, the PCA revealed the real regions structure, Darfour grouped separately in the dendrogram and in the PCA graph. The high proportion of variation attributable to within the region $(80 \%)$, in this study was consistent with the expected population structure for an out crossing species. For pearl millet, it is expected that the heterogeneity and heterozygosity at many loci within a landrace accession to be high. This is due to cross-pollinated nature of the crop. The self-pollinated species such as Hordeum spontaneum has $43 \%$ of within population variation [19]. In contrast, cross-pollinated species has been reported to have $72 \%-100 \%$ of their total variation [20] [21]. Our results are in agreement with the findings of [22], who studied genetic diversity of pearl millet 
in Niger by using AFLP marker. They found that most of the diversity was found at the population level. Also, a previous study done by [23] in India and by [24] in Nigeria reported $88 \%$ to $98 \%$ of the variation was found between individuals within populations. They concluded that the high diversity found within a population may be due to out-breeding reproduction system of pearl millet as well as from the seed flow observed at the regional level. Previous studies on pearl millet from Africa and India based on isozymes diversity indicated intra-population diversity of $70 \%-90 \%$ of the total diversity, depending on their regions of origin [25] [26]. Generally, RAPD technique in this study was very useful. The study provided very useful knowledge about genetic variability of some Pennisetum glaucum accessions from Sudan which may allow more efficient and effective uses of resources in plant improvement programs. In this study, RAPD technique was utilized for better assessment of Pennisetum glaucum accessions relationship collected from four regions of Sudan. The genetic dissimilarity values obtained with RAPD have been introduced for measuring genetic relationships in many plant species for easiness of the method, which only requires PCR technology. This study clearly showed that it was possible to analyze the RAPD and for correlating their similarity and distance between Pennisetum glaucum accessions. From the results of RAPD profiling, it was observed that Pennisetum glaucum accessions produced very good amplification with the primers used. Molecular markers can be used to study the genetic diversity and genetic relationships among Pennisetum glaucum accessions at the DNA level. The present investigation of DNA profiling in Pennisetum glaucum species clearly demonstrates that it is possible to analyze the RAPD patterns for correlating their similarity and distance between accessions. Although accessions from Darfour had the least percentage of polymorphic fragments $(60.3 \%)$ from all the other accessions, they were grouped together in both the dendrogram and the PCA analysis. This shows that, these accessions might maintain alleles (genes) that are unique to the region. North Kordofan had the second least percentage of polymorphic loci (62.9\%). These accessions as well grouped together and were distant from those of Darfour. Although accessions from South Kordofan had the highest percentage of polymorphic loci (88.2\%), they were dispersed with groups of North Kordofan and Blue Nile. The analyses of RAPD revealed high levels of genetic variability, even with the use of limited set of primers. This high level of polymorphism among individuals suggests that RAPD techniques can be useful for Pennisetum glaucum for the maintenance of Germplasm banks and the efficient selection of parents for breeding.

\subsection{Agronomical Analysis}

The results showed that B32 was least similar to others, and seems to be a different millet genotype. S22 was the second least similar genotype to other accessions. From the two dendrograms, the cluster based on data obtained from RAPD markers and the cluster based on the agronomical descriptors, showed no 
similarity between the two results. The accessions were distributed in different sites in both clusters, so the classification based on RAPD markers could not be related to agronomic descriptors about the accessions in this study. These results are in agreement with those results reported by [27] they studied complex samples in two pearl millet fields in two villages of southwestern Niger, by using both morphological and AFLP marker. Also the results are in agreement with [28] they studded diversity in 21 cotton genotypes by using RAPD marker and morphology markers, and [29] they used RAPD marker and morphological marker to study some olive trees. In all previous studies mentioned, there was no co-relation between morphology and genetic data.

The estimates of genetic relationship can be helpful for organizing germplasm, conservation of genetic resources, identification of cultivars for selection of parents for hybridization, and for predicting favorable heterotic combinations. This also helps to reduce the number of samples required for sampling of genetically variable broad range of accessions in breeding programs. Accessions with the most distinct DNA profiles are likely to contain the greatest number of novel alleles, which are likely to uncover the largest number of unique and potentially agronomically useful alleles [27]. However, using both morphological and molecular characterization, provide us with a more complete and informative characterization of the germplasm and this can make breeding programs more easy and useful.

\section{References}

[1] Mhyeldin AIA (2006) Genetic Studies of Pearl Millet (Pennisteum glaucum L.) under Water Stress at Different Growth Stages. M.Sc. Thesis, Agricultural University of Khartoum, Khartoum.

[2] Senthilvel, S., Hash, C.T. and Hoisington, D.A. (2005) Enhancement of the Set of Microsatellite (SSR) Markers of Improving Pearl Millet Breeding Efficiency in Africa and Asia, (ICRSAT), India. Project Concept Note.

[3] Abulgasim, E.H. (1999) A Project for the Multiplication of the Seed Improved and Promising Varieties of Pearl Millet in the Udan. Progress Report.

[4] Hamza, N.B. (2007) Progress and Prospects of Millet Research in Sudan. New Approaches to Plant Breeding of Orphan Crops in Africa, 19-21 September 2007, Bern-Switzer Land, 26.

[5] Briggs, K.G. (1991) Spatial Variation in Seed Size and Seed Set on Spikes of Some Canadian Spring Wheat Cultivars. Canadian Journal of Plant Science, 71, 95-103. https://doi.org/10.4141/cjps91-011

[6] Cadee, N. (2000) Genetic and Environmental Effects on Morphology and Fluctuating Asymmetry in Nestling Barn Swallows. Journal of Evolutionary Biology, 13, 359-370. https://doi.org/10.1046/j.1420-9101.2000.00194.x

[7] Beta, T. and Corke, H. (2001) Genetic and Environmental Variation in Sorghum Starch Properties. Journal of Cerea Science, 34, 261-268.

https://doi.org/10.1006/jcrs.2000.0379

[8] Geleta, N. and Labuschagne, M.T. (2005) Qualitative Traits Variation in Sorghum (Sorghum bicolor (L.) Moench) Germplasm from, Eastern Highlands of Ethiopia. Biodiversity and Conservation, 14, 3055-3064. 
https://doi.org/10.1007/s10531-004-0315-x

[9] Barnaud, A., Deu, M., Garine, E., McKey, D. and Joly, H. (2007) Local Genetic Diversity of Sorghum in a Village in Northern Cameroon: Structure and Dynamics of Landraces. Theoretical and Applied Genetics, 114, 237-248. https://doi.org/10.1007/s00122-006-0426-8

[10] Smith, J.S.C. and Smith, O.S. (1992) Fingerprinting Crop Varities. Advances in Agronomy, 47, 85-140. https://doi.org/10.1016/S0065-2113(08)60489-7

[11] Redfearn, D.D., Buxton, D.R. and Devine, T.E. (1999) Sorghum Intercropping Effects on Yield, Morphology, and Quality of Forage Soybean. Crop Science, 39, 1380-1384. https://doi.org/10.2135/cropsci1999.3951380x

[12] Antunes, M.S., Vasconcelos, M.J.V. and Nett, D.A.M. (1997) RAPD Analysis of Pearl Millet Cultivars. International Sorghum and Millet News Letter, 38, 146-150.

[13] Porebski, S., Bailey, L.G. and Baum, R. (1997) Modification of a CTAB DNA Extraction Protocol for Plants Containing High Polysaccharide and Polyphennol Components. Plant Molecular Biology Reporter, 15, 8-15.

https://doi.org/10.1007/BF02772108

[14] Nei, M. and Li, W. (1979) Mathematical Model for Studying Genetic Variation in Terms of Restriction Endonucleases. Proceedings of the National Academy of Sciences of the United States of America, 76, 5269-5273.

https://doi.org/10.1073/pnas.76.10.5269

[15] Peakall, R. and Smouse, P.E. (2006) Genealex 6: Genetic Analysis in Excel. Population Genetic Software for Teaching and Research. Molecular Ecology Notes, 6, 288-295. https://doi.org/10.1111/j.1471-8286.2005.01155.x

[16] Hauser, L.A. and Crovello, T.J. (1982) Numerical Analysis of Genetic Relationships in Thelypodieae (Brassicaceae). Systematic Botany, 7, 249-268. https://doi.org/10.2307/2418387

[17] StatSoft Inc. (2001) STATISTCA (Data Analysis Software System), Version 6.

[18] SPSS (1995) Computer Users, Guide SPSS in. USA StatSoft lnc. 2001 STATISTCA (Data Analysis Software System), Version 6.

[19] Dawson, I.K., Chalmers, K.J., Waugh, R. and Powell, W. (1993) Detection and Analysis of Genetic Variation in Hordeum spontaneum Population from Israel Using RAPD Markers. Molecular Ecology, 2, 151-159. https://doi.org/10.1111/j.1365-294X.1993.tb00104.x

[20] Huff, D.R., Peakell, R. and Smonse, P.E. (1993) RAPD Variation within and between Natural Populations of Out-Crossing Buffalo Grass (Buchloe dactyloides (Nutt). Englem.). Theoretical and Applied Genetics, 86, 927-934. https://doi.org/10.1007/BF00211043

[21] Nesbitt, K.A., Potts, B.M., Vaillan Court, R.E., West, A.K. and Reid, J.B. (1995) Partitioning and Distribution of RAPD Variation in a Forest Tree Species, Eucalyptus globulus (Myrtaceae). Heredity, 74, 628-637. https://doi.org/10.1038/hdy.1995.86

[22] Allinne, C., Mariac, C., Vigouroux, Y., Bezençon, G., Couturon, E., Moussa, D., Tidjani, M., Pham, J.-L. and Robert, T. (2008) Role of Seed Flow on the Pattern and Dynamics of Pearl Millet (Pennisetum glaucum [L.] R. Br.) Genetic Diversity Assessed by AFLP Markers: A Study in South-Western Niger. Genetica, 133, 167.

[23] Vom Brock, K., Christin, C.K.A., Welt Zien, E.R., Prester, I.T. and Geiger, H.H. (2003) Farmer's Seed Systems and Management Practices Determine Pearl Millet Genetic Diversity Patterns in Semi Arid Regions of India. Crop Science, 43, 
1680-1689. https://doi.org/10.2135/cropsci2003.1680

[24] Busso, C.S., Devos, K.M., Ross, G., Mortimore, M., Adams, W.M., Ambrose, M.J., Alldrick, S. and Gale, M.-D. (2000) Genetic Diversity within and among Landraces of Pearl Millet (Pennisetum glaucum) under Farmer Management in West Africa. Genetic Resources and Crop Evolution, 47, 561-568.

https://doi.org/10.1023/A:1008767220320

[25] Tostain, S., Riandey, M.F. and Marchais, L. (1987) Enzyme Diversity in Pearl Millet (Pennisetum glaucum). 1 West Africa. Theoretical and Applied Genetics, 74, 188-193. https://doi.org/10.1007/BF00289967

[26] Tostain, S. and Marchais, L. (1989) Enzyme Diversity I Pearl Millet (Pennisetum glaucum). 2 Africa and India. Theoretical and Applied Genetics, 77, 634-640. https://doi.org/10.1007/BF00261235

[27] Mariac, C., Robert, T., Allinne, C., Remigereau, M.S., Luxereau, A., Tidjani, M., Seyni, O., Bezancon, G., Pham, J.L. and Sarr, A. (2006b) Genetic Diversity and Gene Flow among Pearl Millet Crop/Weed Complex: A Case Study. Theoretical and Applied Genetics, 113, 1003-1014. https://doi.org/10.1007/s00122-006-0360-9

[28] Esmail, R.M., Zhang, J.F. and Abdel-Hamid, A.M. (2008) Genetic Diversity in Elite Cotton Germplasm Lines Using Field Performance and RAPD Markers. World Journal of Agricultural Sciences, 4, 369-375.

[29] Ozkaya, M.T., Cakir, E., Gokbayrak, Z., Ercan, H. and Taskin, N. (2006) Morphological and Molecular Characterization of Derik Halhali Olive (Olea europaea L.) Accessions Grown in Derik-Mardin Province of Turkey. Scientia Horticulture, 108, 205-209. https://doi.org/10.1016/j.scienta.2006.01.016 